Acta Crystallographica Section A

Foundations of Crystallography

ISSN 0108-7673

Received 15 June 2007

Accepted 27 August 2007

\section{The interpretation and analysis of diffuse scattering using Monte Carlo simulation methods}

\author{
T. R. Welberry* and D. J. Goossens
}

Research School of Chemistry, Australian National University, Canberra, ACT 0200, Australia. Correspondence e-mail: welberry@rsc.anu.edu.au

\begin{abstract}
Studies of diffuse scattering had a prominent place in the first issue of Acta Crystallographica 60 years ago at a time when conventional crystallography (determination of the average structure from Bragg peaks) was in its infancy. Since that time, conventional crystallography has developed enormously while diffuse-scattering analysis has seemingly lagged well behind. The paper highlights some of the extra difficulties involved in the measurement, interpretation and analysis of diffuse scattering and plots the progress that has been made. With the advent of the latest X-ray and neutron sources, area detectors and the ever-increasing power of computers, most disorder problems are now tractable. Two recent contrasting examples are described which highlight what can be achieved by current methods.
\end{abstract}

(C) 2008 International Union of Crystallography Printed in Singapore - all rights reserved time. It is salutary to realize that, at that stage, it was still common practice for small-molecule crystal structures to be determined by considering only two-dimensional reciprocallattice sections, with atom positions being located from Fourier maps of the corresponding structure projections (Abrahams \& Robertson, 1948; Broomhead \& Nicol, 1948; Jerslev, 1948). Two such sections were sufficient to determine $x, y$ and $z$ atomic coordinates. The Cambridge Structural Database (Allen, 2002) and the Protein Data Bank (Berman et al., 2000) bear witness to the enormous progress that has been made in conventional crystallography since that time.

The question may, therefore, be asked: why hasn't there been the same progress in the analysis of diffuse scattering as has taken place in conventional crystallography? There are two main reasons. Firstly, diffuse-scattering intensities are very weak in comparison to Bragg peaks, making the experimental observation vastly more demanding and time consuming. Only with the increasing availability of synchrotron radiation has this obstacle been, if not removed, substantially diminished. Secondly, the sheer diversity of different types of disorder and their effect on the diffraction pattern made it difficult to formulate a solution strategy that would work for all problems. Substantial progress was made relatively early on in some specific areas. For example, in disordered alloys and simple oxides, quantitative methods evolved in which use was made of large samples and only restricted regions of reciprocal space needed to be measured. Only relatively recently has the rapid advance in computing power (processor speeds, memory and storage) made it possible for computer simulation of a model system to emerge as a general method by which virtually any diffuse-scattering problem can be tackled in a systematic manner.

In answer to the above question, it may be said that there has been a great deal of progress in the measurement, 
interpretation and analysis of diffuse scattering but this has taken place starting from a much lower base line than that for conventional crystallography. In this paper, we describe the progress that has been made and show with the aid of some recent examples the current state of the methodology. It will be argued that the analysis of diffuse scattering today has reached a point in its development similar to that reached by conventional crystallography in 1948 and is, analogously, poised to enter a period of great expansion and exploitation.

\section{Experimental}

\subsection{Measurement of $X$-ray diffuse scattering}

Although, as mentioned above, diffuse scattering has been observed and studied from the earliest days of X-ray crystallography, obtaining high-quality quantitative diffuse-scattering data has been far from routine and this has been a major impediment to progress in the field until relatively recently. The actual intensities involved are typically $\sim 10^{3}-10^{4}$ down on Bragg-peak intensities. Consequently, if a single-point detector is used and measurements are made at increments of say 0.1 of the reciprocal-lattice repeats (a bare minimum requirement), this already represents a factor of $\sim 10^{6}-10^{7}$ increase in counting time compared with a Bragg experiment. Even so, the intensities that are available from the latest generation synchrotron sources, together with the latest multidetectors (wire-based gas detectors, image plates or CCD detectors etc.), are more than sufficient to compensate for this disparity in magnitude and it is now quite feasible to obtain high-quality three-dimensional diffuse X-ray scattering data in a relatively routine manner. Commonly used is the so-called Laue method in which the incident beam of monochromatic $\mathrm{X}$-rays is directed onto a stationary crystal. The Ewald sphere intersects the diffuse features in reciprocal space, producing a continuous distribution of intensity in the scattered pattern. This is then recorded on a two-dimensional flat-plate detector placed behind the crystal, as shown in Fig. 1(a). Note that with a stationary crystal the Ewald sphere will not pass directly through any of the Bragg peaks except by chance so the scattered intensity in a given frame shows only diffuse peaks with the occasional accidental Bragg peak. This method closely follows that adopted in early studies of diffuse scattering, e.g. Lonsdale (1948a), Flack (1970) and Glazer (1970), though there the characteristic radiation from a tube source was used and the diffraction patterns were contaminated with many Bragg peaks resulting from the the white radiation (Bremsstrahlung) present in the source. Fig. 1(b) shows a single frame obtained at beamline 1-ID-C at the Advanced Photon Source (APS) for the material pentachloronitrobenzene (PCNB), see $\$ 3$. The exposure here was $2 \mathrm{~s}$ compared with the many minutes or even hours that were required in those early studies.

In order to obtain a complete three-dimensional volume of diffuse-scattering data, a large number of frames are recorded as the crystal is rotated incrementally in steps of $\Delta \omega$ (typically by 0.25 or $0.36^{\circ}$ ). From this three-dimensional volume of scattering, any desired two-dimensional plane section can then be extracted using appropriate software [e.g. the program EXCAVATE (Estermann \& Steurer, 1998)]. An alternative but only slight modification of the above method is sometimes used in which the crystal is rotated through the $\Delta \omega$ increment during exposure. This has little effect on the resolution of the diffuse scattering but means that all the Bragg peaks will be included in the data. Although the above process is capable of yielding high-quality diffuse-scattering data, the low signal level of diffuse scattering still presents considerable experimental challenges compared with Bragg scattering. It is crucial to avoid or account for the various forms of parasitic scattering that may be present in experiments: e.g. air scattering, stray scattering from collimators, windows, capillaries, beam stops etc. These may be of a comparable magnitude to the diffuse-scattering signal being measured and, since they mainly arise from the incident beam or the Bragg peaks

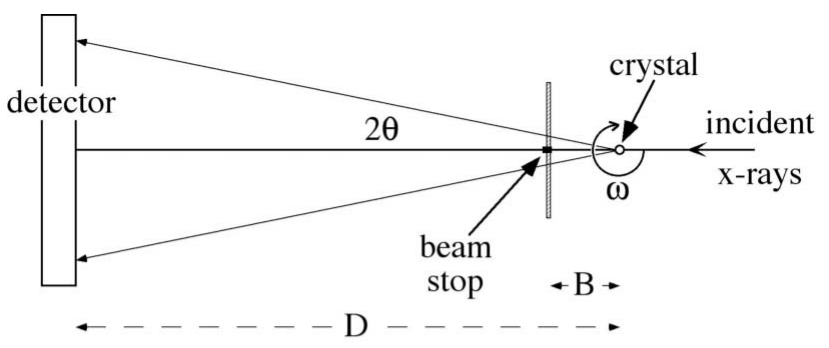

(a)

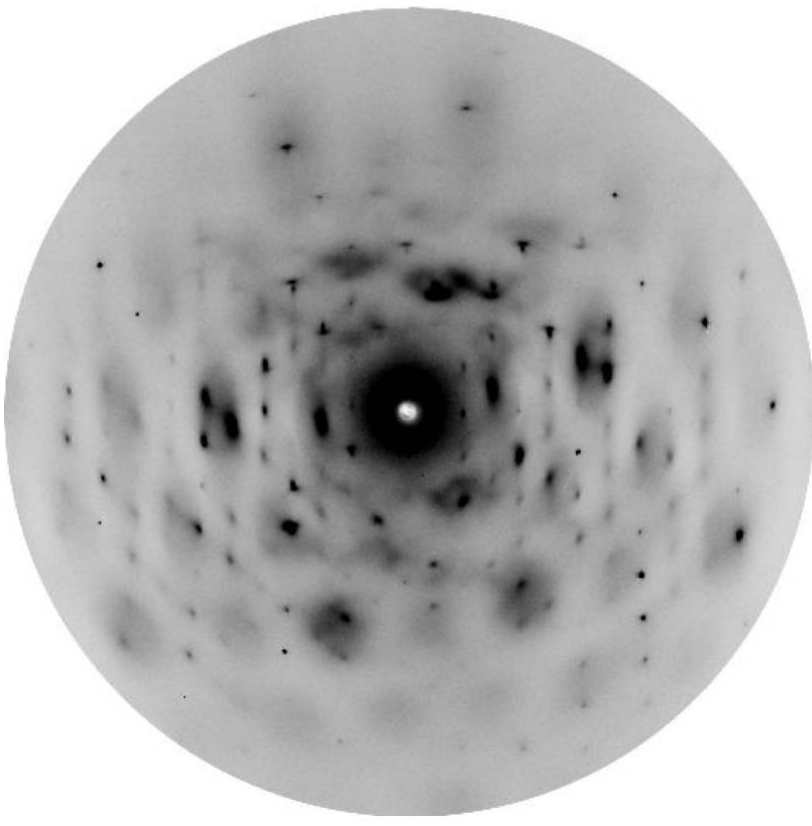

(b)

Figure 1

(a) The experimental arrangement used to record diffuse scattering using high-energy X-rays $(\lambda=0.30996 \AA)$. (b) A single frame of data for PCNB recorded with a $2 \mathrm{~s}$ exposure using a Mar345 Image-plate detector. 500 frames, recorded at intervals of $0.36^{\circ}$ of the crystal rotation, $\omega$, were used for the complete three-dimensional set of data used in the study described in $\$ 3$. The dark region near the middle of the pattern is due to air scattering. This was subsequently subtracted during processing. The maximum $\sin \theta / \lambda$ recorded was $0.85 \AA^{-1}$. 
themselves, they remain a problem even with synchrotron radiation (Welberry, Goossens et al., 2005). Meticulous attention to detail is required in experiments to avoid or overcome them.

\subsection{Measurement of neutron diffuse scattering}

The neutron fluxes available at the best neutron facilities in the world (there were very few in 1948) are so low in comparison to the photon fluxes available from synchrotrons that it might be supposed that obtaining diffuse-scattering data using neutrons is simply not viable, except perhaps for small selected regions of reciprocal space. This is in fact not the case and for suitable samples valuable three-dimensional diffuse-scattering data can be obtained in a relatively short time. Whereas for X-ray diffuse scattering a single wavelength must be used so that most of the available X-rays are discarded, for neutrons produced at a spallation source it is possible to utilize the whole spectrum of incident neutron wavelengths and separate their contributions to the scattering via time-of-flight spectroscopy. This in itself provides a large enhancement factor compared with experiments relying on only a single wavelength. When coupled with the possibility of surrounding the sample with detector banks, which cover a large fraction of the complete solid angle, data acquisition rates sufficient to allow the measurement of diffuse-scattering data can be achieved for a reasonably sized sample $(<10 \mathrm{~mm}$ linear dimension). In $\$ 4$, we describe a study using diffuse neutron data recorded at the SXD single-crystal beamline at ISIS, Rutherford Appleton Laboratory, UK. This instrument has 11 detector banks, together covering close to $50 \%$ of the complete solid angle surrounding the sample. Each of the detectors comprises $64 \times 64$ pixels and each pixel records a complete time-of-flight spectrum. This gives a formidable amount of data simultaneously from a single setting of the sample. The new single-crystal instrument TOPAZ currently being developed at SNS in Oakridge, Tennessee, USA, is expected to improve on the performance of SXD by a significant factor, both in total flux and in resolution and will be ideally suited to this kind of experiment.

\subsection{Interpretation and analysis}

By far the largest impediment to the utilization of the rich source of information that diffuse scattering affords is the diversity and often complex combination of disorder effects that arise in nature and the fact that until recently no one simple method of analysis has been available which can usefully deal with all of them. Much of the early work on diffuse scattering was confined to the area of metal alloys and simple oxides (Borie \& Sparks, 1971; Tibballs, 1975; Georgopoulos \& Cohen, 1977) and these methods, though well developed, were not easily transferable to more general systems and problems. Our aim has been to develop methods to make the analysis of diffuse scattering more routine and applicable to a wider and more diverse range of different materials. To this end, we have developed the use of largescale Monte Carlo (MC) computer simulations and these have been used for a wide range of materials including complex oxides, other ceramics and inorganic materials, organic molecular crystals, inclusion compounds and even quasicrystals.

Monte Carlo computer simulation of a model structure has become a powerful and well accepted technique for aiding the interpretation and analysis of diffuse-scattering patterns (Weber et al., 2001; Weber \& Bürgi, 2002; Welberry, 2004). In this method, a computer model is set up based on known physical and chemical principles with adjustable parameters describing the basic interatomic or inter- and intramolecular interactions and possible mechanisms for disorder. Diffraction patterns are then calculated from the results of the simulations and these are compared with the observed data. As a result of the comparison, adjustments are made to the model parameters and the process is repeated until agreement between observed and calculated patterns is obtained. By use of such MC simulations, different models can be tested. Those that are incorrect can be definitely eliminated while substantial confidence can be placed in a model that gives good agreement with the observation, since reliable physical and chemical principles have been built in at the outset. The advantage of the method is that it can be applied generally to all systems regardless of their complexity or the size of the atomic displacements that might be present. The only limitation is the extent to which the MC energy can be made to realistically represent the real system energy. At one extreme, a very simplified model may be useful in demonstrating particular qualitative effects (Welberry, 2001) while, at the other, a quantitative and very detailed description of a disordered structure can be obtained (Welberry et al., 2001).

In the following sections, we describe two contrasting recent examples to show what can be achieved with the latest techniques. One example is of a disordered organic material and the other an inorganic ceramic material. In the first case, the simulation model results are compared with X-ray diffuse scattering and, in the second, the comparison is with neutron diffuse scattering.

\section{Example 1: pentachloronitrobenzene, $\mathrm{C}_{6} \mathrm{Cl}_{5} \mathrm{NO}_{2}$}

In this section, we discuss a recent example which has been described in detail in Thomas et al. (2007). Pentachloronitrobenzene (PCNB) is one of a series of chloronitrobenzene derivatives that are currently being studied because their disordered nature and propensity to undergo phase transitions are thought to be responsible for their anomalous dielectric properties (Hall \& Horsfall, 1973). Disorder often strongly affects the electronic environment in such materials, and it is considered that this may be fundamental to physical properties such as second-harmonic generation (Cole et al., 2000).

Conventional crystallographic studies of PCNB (Thomas, 2007; Cole et al., 2007) have revealed that each molecular site in PCNB contains the molecule in one of six different orientations each with equal probability of occurrence, giving the 
molecular site full sixfold symmetry on average. Fig. 2 shows drawings of the average structure. The diffraction patterns of PCNB show strong highly structured diffuse scattering, see Fig. 3. For further details see Cole et al. (2007).

\subsection{Monte Carlo simulation}

Monte Carlo simulation was carried out as a two-stage process. In the first stage, the possibility of occupational ordering was investigated. A MC energy was used which depended on the mutual orientation of neighbouring pairs of

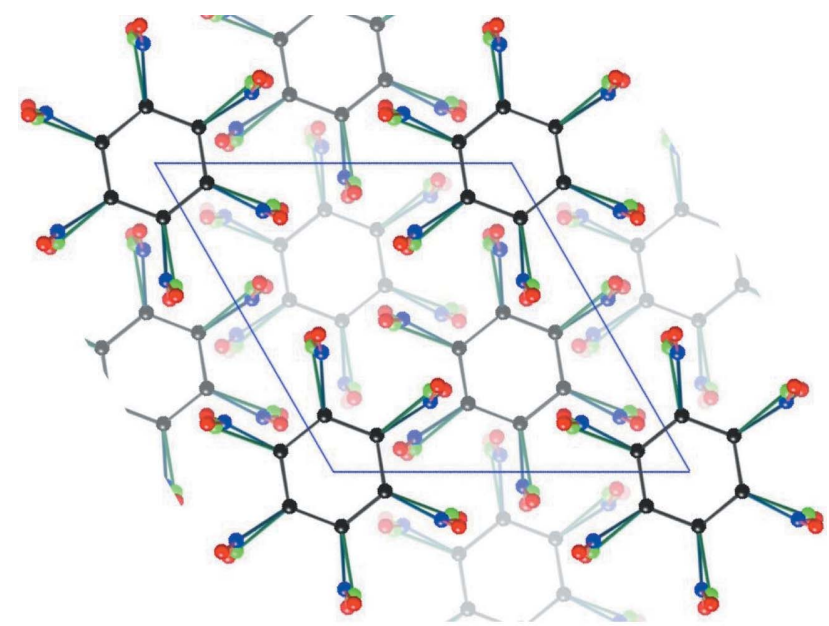

(a)

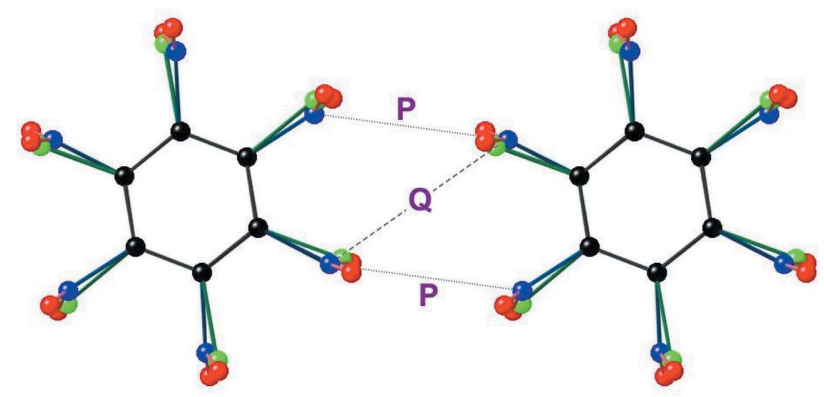

(b)

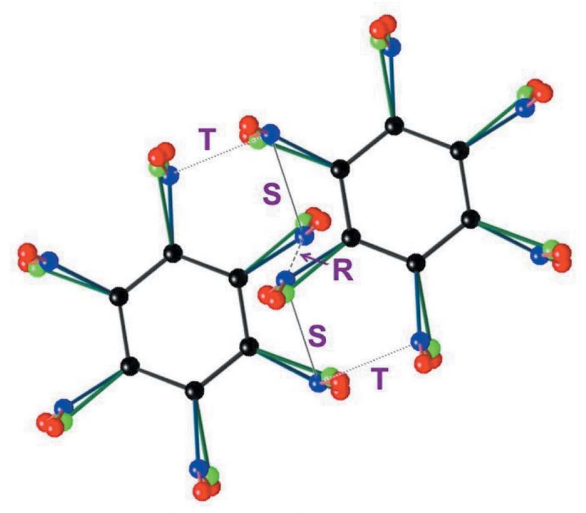

(c)

\section{Figure 2}

(a) The view down [001] of the average structure of PCNB. Each of the six substituent sites in every molecular site contains $\frac{1}{6} \mathrm{NO}_{2}$ and $\frac{5}{6} \mathrm{Cl}$. (b), (c) The five different interaction vectors used in the MC simulation: $(b)$ within the molecular planes, $(c)$ between neighbouring molecular planes. molecules, both for intermolecular vectors within molecular planes normal to [001] and for vectors between planes. Since each molecular site had the possibility of one of six different orientations resulting in 36 possible different mutual orientations, this part of the study involved exploration of a

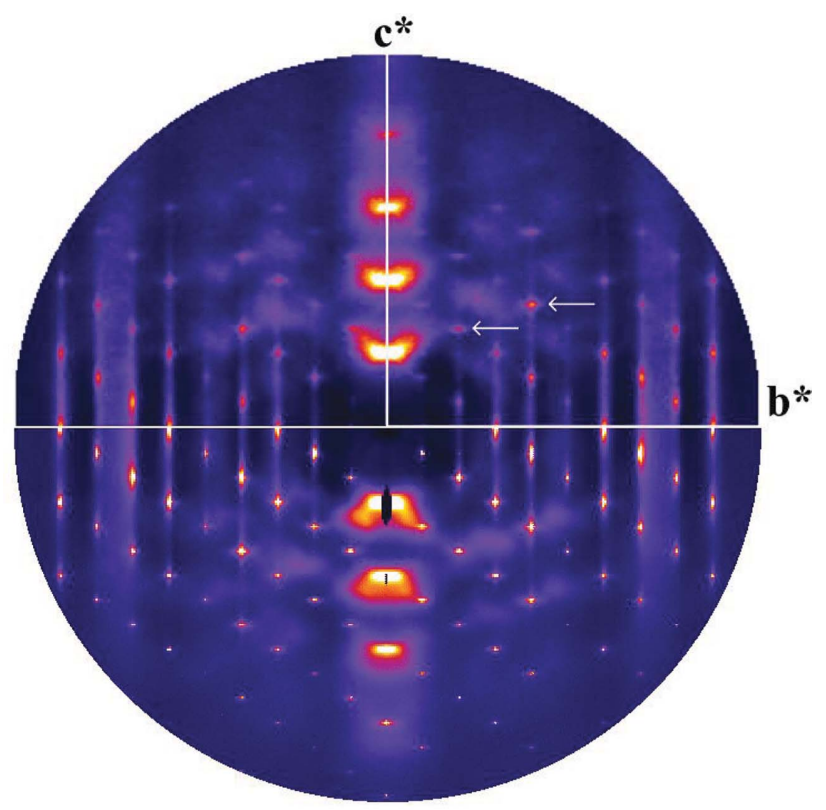

(a)

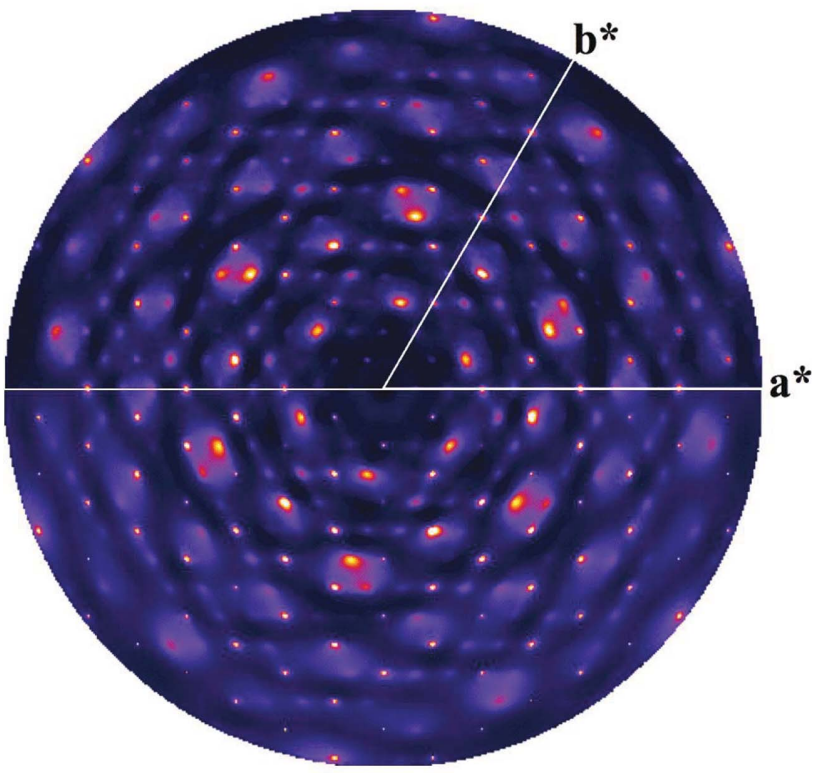

(b)

0 $\max$

Figure 3

Calculated diffuse-scattering patterns (upper semicircles) for PCNB compared with the corresponding observed X-ray data (lower semicircles). (a) $0 \mathrm{kl}$ section. (b) $h k 0$ section. (Note the upper and lower halves are not related by $m$ symmetry. $0 \mathrm{kl}$ has twofold symmetry; $h k 0$ has sixfold symmetry. It should also be noted that the calculated patterns contain no actual Bragg peaks since the average structure is subtracted during the calculation.) 
Table 1

The force constants, $k_{i}$, and size-effect parameters, $\varepsilon_{i}$, used for the simulation whose diffraction patterns are shown in Fig. 3.

The $k_{i}$ are in units of $k T$ and the $\varepsilon_{i}$ are in $\AA$. Note that for each vector the four values of $\varepsilon_{i}$ are subject to three constraints so that they represent only one independent parameter.

\begin{tabular}{lcrccc}
\hline Vector type & \multicolumn{1}{c}{$k_{i}$} & \multicolumn{1}{c}{$\varepsilon^{\mathrm{NN}}$} & $\varepsilon^{\mathrm{NCl}}$ & $\varepsilon^{\mathrm{ClN}}$ & $\varepsilon^{\mathrm{ClCl}}$ \\
\hline$P$ & 3.94 & 0.0 & 0.0 & 0.0 & 0.0 \\
$Q$ & 3.94 & 0.0 & 0.0 & 0.0 & 0.0 \\
$R$ & 2.62 & +1.5 & +0.75 & +0.75 & -0.36 \\
$S$ & 2.62 & +2.0 & +1.0 & +1.0 & -0.48 \\
$T$ & 1.31 & 0.0 & 0.0 & 0.0 & 0.0 \\
\hline
\end{tabular}

multidimensional parameter space. However, after extensive searching, it was concluded that of the many possible ordering patterns none was consistent with the observed diffraction patterns and that the occupancy of a given site was therefore essentially random. Hence the observed scattering must originate almost entirely from the displacements induced by the different sizes of the $\mathrm{NO}_{2}$ and $\mathrm{Cl}$ groups.

The second stage of MC simulation was carried out with this random occupation model. In this second-stage model, the basic molecular orientations were maintained but were, together with the centre-of-mass coordinates, subject to local perturbations induced by the molecular neighbourhood. Each molecule was treated as a rigid unit and intermolecular interactions were represented by harmonic (Hooke's law) springs, both between nearest-neighbouring molecular sites within the molecular planes normal to [001] and between nearest-neighbour molecules in adjacent layers. These two different intermolecular interactions were comprised of five interatomic interactions (springs) labelled $P, Q, R, S$ and $T$ as shown in Figs. $2(b)$ and $2(c)$. The MC energy, $E_{\text {disp }}$, which allowed for displacements due to thermal motion and size effects, was of the form

$$
E_{\text {disp }}=\sum_{i=1}^{5} k_{i} \sum_{\text {types } a b}\left(d-d_{i}^{a b}\right)^{2} .
$$

The spring constants, $k_{i}$, associated with the five types of vector were treated as parameters to be determined in the analysis.

In equation (1), $d$ is the instantaneous length of a particular interatomic vector. $d_{i}^{\mathrm{ab}}$ are equilibrium spring lengths which depend not only on the type of contact, $i$, but also on the particular pair of atoms, $a b$, that take part in the contact, i.e. $a b$ may be $\mathrm{NN}, \mathrm{NCl}, \mathrm{ClN}$ or $\mathrm{ClCl}$. These values of the spring lengths are defined relative to the vector distances, $l_{i}^{a b}$, observed in the average structure.

$$
d_{i}^{a b}=l_{i}^{a b}\left(1+\varepsilon_{i}^{a b}\right) .
$$

$\varepsilon_{i}^{a b}$ are size-effect parameters which describe the local perturbations due to the differences in size of the $\mathrm{NO}_{2}$ and $\mathrm{Cl}$ groups. Although for each type of vector there are four different values, $\varepsilon_{i}^{a b}$, these are subject to constraints in order that the average lengths $\left(l_{i}^{a b}\right)$ are maintained and only a single independent value, $\varepsilon_{i}$, was used for each vector type $i$. In all,
Table 2

The effect of size on atom-atom vectors comprising the intermolecular vector $R$.

See Fig. 2. Distances given are in $\AA$.

\begin{tabular}{llllrr}
\hline Vector type & $\begin{array}{l}\text { Average } \\
\text { structure }\end{array}$ & Mean & $\sigma$ & $\Delta d$ & \% change \\
\hline $\mathrm{N} \cdots \mathrm{N}$ & 3.817 & 4.404 & 0.27 & 0.587 & +15 \\
$\mathrm{~N} \cdots \mathrm{Cl}$ & 3.919 & 4.201 & 0.27 & 0.282 & +7 \\
$\mathrm{Cl} \cdots \mathrm{N}$ & 3.919 & 4.201 & 0.27 & 0.282 & +7 \\
$\mathrm{Cl} \cdots \mathrm{Cl}$ & 4.035 & 3.951 & 0.27 & -0.084 & -2 \\
\hline
\end{tabular}

five independent spring constants, $k_{i}$, together with a single size-effect parameter, $\varepsilon_{i}$, associated with each were the only parameters used in the analysis.

\subsection{Results}

The MC simulations were carried out on a model crystal comprising $48 \times 48 \times 48$ unit cells and 1000 cycles of iteration were used in order to allow the system to closely approach equilibrium. Values of the parameters described above were adjusted to give the best fit to the observed data. The final values are given in Table 1 . These values indicate that nonzero values of $\varepsilon_{i}$ were only given to the vectors $R$ and $S$ which are involved in the intermolecular contact between molecular layers. Fig. 3 shows a comparison of the observed and calculated diffraction patterns obtained from this model for two different sections. There is clearly excellent agreement between the observed and calculated patterns.

Fig. 4 shows scatter plots of the distribution of atomic positions in the molecular sites at $\frac{1}{3} c$ and $\frac{2}{3} c$. The figure on the left includes all unit cells in the structure. That on the right shows only those unit cells in which the two sites are both occupied by molecules with $\mathrm{NO}_{2}$ in the terminal position. For these molecules, the $\mathrm{N} \cdots \mathrm{N}$ intermolecular vector is increased by $\sim 0.6 \AA$ relative to the average, resulting in a tilting of the molecular planes. When the two terminal positions are both occupied by $\mathrm{Cl}$, the $\mathrm{Cl} \cdots \mathrm{Cl}$ vector is slightly less than average (this occurs more frequently). For one position occupied by $\mathrm{Cl}$ and the other by $\mathrm{NO}_{2}$, the $\mathrm{NO}_{2} \cdots \mathrm{Cl}$ vector is increased by

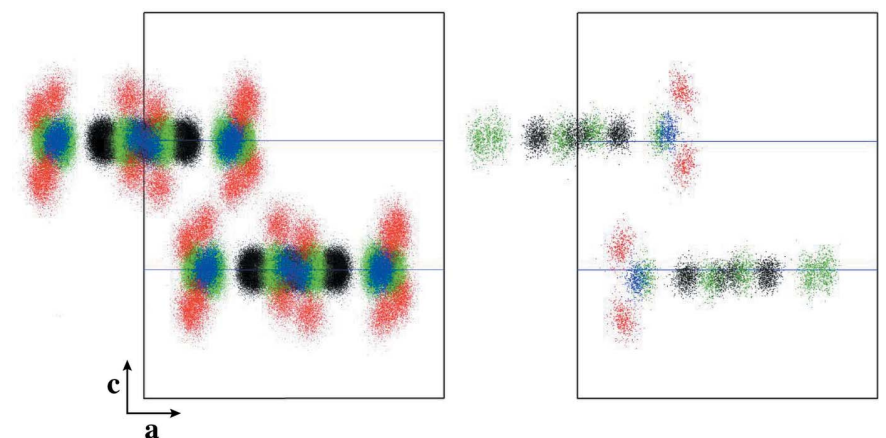

Figure 4

Scatter plots for PCNB showing the distribution of atomic positions in the molecular sites at $\frac{1}{3} c$ and $\frac{2}{3} c$. Red is $\mathrm{O}$ atoms; blue is $\mathrm{N}$ atoms; green is $\mathrm{Cl}$ atoms; black is $\mathrm{C}$ atoms. See text for details. 
$\sim 0.3 \AA$. These figures are summarized in Table 2 . These results show that the strong, detailed and very distinctive diffraction patterns observed for PCNB can be accounted for almost entirely on the assumption of random occupancy of each molecular site but with very large local relaxation displacements that tend to increase the neighbouring distances for contacts involving $\mathrm{NO}_{2} \cdots \mathrm{NO}_{2}$ (by $0.6 \AA$ ) and $\mathrm{NO}_{2} \cdots \mathrm{Cl}$ (by $0.3 \AA$ ) with a corresponding reduction for those involving $\mathrm{Cl} \cdots \mathrm{Cl}$.

The magnitude of these displacements are very large when compared with the basic spacing of the intermolecular layers (3.75 ̊). To achieve these levels of distortion, the molecules must be very uncomfortable in the average structure geometry. It is therefore surprising that the system chooses to pack this way rather than find an energy minimum defining a different crystal structure which will accommodate the differently sized substituents in a more ordered arrangement that does not require such large local strain.

\section{Example 2: polar nanodomains in $\mathrm{PbZn}_{1 / 3} \mathrm{Nb}_{2 / 3} \mathrm{O}_{3}$}

In this section, we discuss a recent example which has been described in detail by Welberry et al. (Welberry, Gutmann et al., 2005; Welberry et al., 2006). Relaxor ferroelectrics have attracted tremendous attention in recent years because of their extraordinary dielectric and piezoelectric properties (Park \& Shrout, 1997). Of these materials, the lead-based perovskite relaxor, $\mathrm{Pb}\left(\mathrm{Zn}_{1 / 3} \mathrm{Nb}_{2 / 3}\right) \mathrm{O}_{3}(\mathrm{PZN})$ has been one of the most extensively studied (Ye et al., 1999; Bing et al., 2005). Despite this, the exact nature of the structure and how it relates to its relaxor properties is still the subject of much controversy. It is generally accepted that, well above the Curie temperature, the structure of PZN and similar relaxors is comprised of nanometre-sized polar domains or nano-regions (PNRs) (see for example Burns \& Dacol, 1983) and it is widely believed that these play an essential role in the relaxor properties (Cross, 1987). Although their existence is well accepted, their structure and origin are still the subjects of controversy. For example, it is accepted that a key feature is that the $\mathrm{Pb}^{2+}$ ions are displaced from the average site position at the centre of the coordination shell of $\mathrm{O}$ atoms, but there is little consensus as to whether the displacements are in the $\langle 100\rangle$ directions (Dmowski et al., 2000), the $\langle 111\rangle$ directions (Woodward \& Baba-Kishi, 2002) or the $\langle 110\rangle$ directions (Welberry, Gutmann et al., 2005). There is also controversy concerning the presence or otherwise of $B$-cation order and whether the $\mathrm{Pb}^{2+}$ ion displacement order is directly related to this. Diffuse scattering is one of the few techniques that is able to probe such local structural detail and analysis using MC simulation techniques can resolve these issues (Welberry et al., 2006).

\subsection{Neutron diffuse-scattering data}

Fig. 5 shows diffraction patterns for three sections of neutron diffuse-scattering data for PZN taken from a
Table 3

Definition of the $n$ basic displacements along $x, y$ and $z$ for the 12-state vector quantity $\Omega_{n}$ representing the $\mathrm{Pb}$ ions.

\begin{tabular}{rrrrrrrrrrrrr}
\hline$n$ & 1 & 2 & 3 & 4 & 5 & 6 & 7 & 8 & 9 & 10 & 11 & 12 \\
\hline$x$ & 0 & 0 & 0 & 0 & $\delta$ & $\delta$ & $-\delta$ & $-\delta$ & $\delta$ & $\delta$ & $-\delta$ & $-\delta$ \\
$y$ & $\delta$ & $\delta$ & $-\delta$ & $-\delta$ & 0 & 0 & 0 & 0 & $\delta$ & $-\delta$ & $\delta$ & $-\delta$ \\
$z$ & $\delta$ & $-\delta$ & $\delta$ & $-\delta$ & $\delta$ & $-\delta$ & $\delta$ & $-\delta$ & 0 & 0 & 0 & 0 \\
\hline
\end{tabular}

complete three-dimensional volume recorded on the SXD beamline at ISIS, Rutherford Appleton Laboratory, UK. The lower half of each frame is the observed data. These show that the scattering is comprised of a number of distinctive features, which any model of the system must be able to explain satisfactorily. The most significant features of the $(h k 0)$ and $(h k 1)$ sections are the diffuse lines ('rods' in three dimensions) which run along rows of Bragg peaks in $\langle 110\rangle$ directions. The diffuse lines show strong azimuthal variation clearly indicating that those normal to [110] are the result of atomic displacements in the [110] direction (and, similarly, those normal to [110] are the result of atomic displacements in the [110] direction). A second most significant feature of the patterns is the fact that the diffuse lines in the $(h k 1)$ section are virtually extinct for $h+k=n$, with $n$ even. Those in the $(h k 0)$ section, however, occur for $n$ both odd and even.

A third important feature is that there are two different kinds of diffuse spots that occur in the $\left(h k \frac{1}{2}\right)$ section, Fig. 5(c). The spots occur in columns for $h+l=n$, or in rows for $k+l=n$, for $n$ integral. One type of spot results from the intersection of diffuse rods related by symmetry to those appearing in the $(h k 0)$ and $(h k 1)$ sections. For example, along the column of diffuse spots at $h=\frac{5}{2}, l=\frac{1}{2}$ in Fig. 5(c), those spots for which $k$ is integral derive from the diffuse rods. The spots for which $k+\frac{1}{2}$ is integral are not due to the diffuse rods and must derive from a different source. In fact, it can be shown that these are due to local ordering of the perovskite $B$-site cations.

Finally, there is evidence in all three patterns of local 'sizeeffect' distortions. These are manifest in the asymmetric shapes of the strong diffuse peaks that surround the Bragg positions and also in the displacement of the diffuse peaks in Fig. 5(c) away from, for example, the exact $h=\frac{5}{2}$ or $h=\frac{7}{2}$ positions.

\subsection{Monte Carlo simulation}

The $\langle 110\rangle$ reciprocal-space rods of scattering discussed above clearly point to the presence of some kind of planar defect in the real-space structure. Such planes must exist normal to each of the six $\langle 110\rangle$ directions. In addition, the azimuthal variation of intensity associated with each set of $\langle 110\rangle$ rods indicates that the dominant atomic displacements must lie within this plane and also be directed along a $\langle 110\rangle$ direction. For example, for the rods that exist parallel to [11 0$]$, the plane which gives rise to this contains the vectors [001] and 
[110], and the displacements must be directed predominantly along [110].

The absence of the $h+k=n, n$ even, rods in ( $h k 1)$ (Fig. $5 b)$ is a kind of selection rule which results if the displacement of an atom at the origin (i.e. $\mathrm{Pb}$ ) is correlated to the displacement of an atom at the centre of the unit cell (i.e. $\mathrm{Nb} / \mathrm{Zn}$ ). However, such a correlation would also result in the absence of the $h+k=n, n$ odd, rods in the ( $h k 0)$ section (Fig. $5 a)$. In order to break this selection rule for $(h k 1)$ and not for $(h k 0)$, it is necessary that the $\mathrm{O} 1$ ions move in the opposite direction to the cations. These different requirements were put together in formulating a simple displacement pattern structure that is

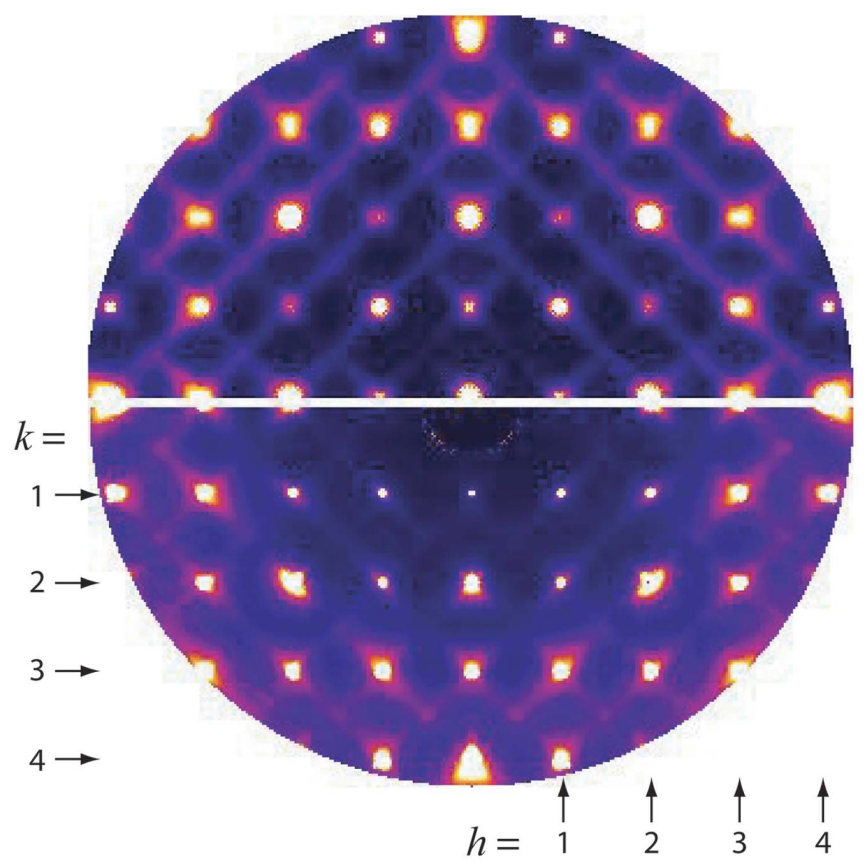

(a)

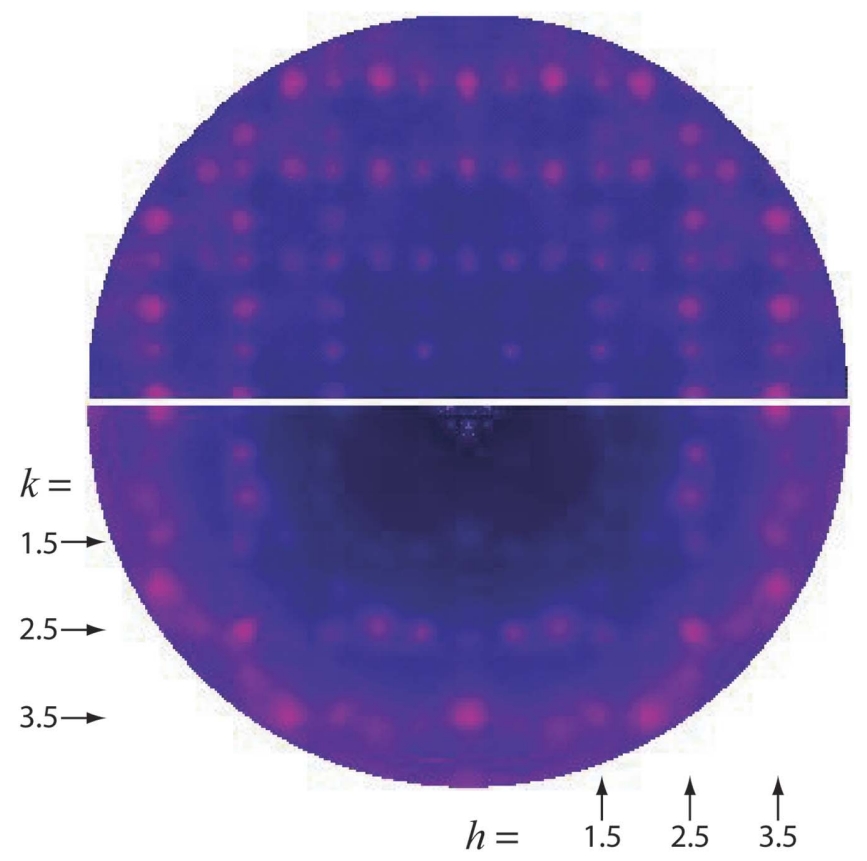

(c)

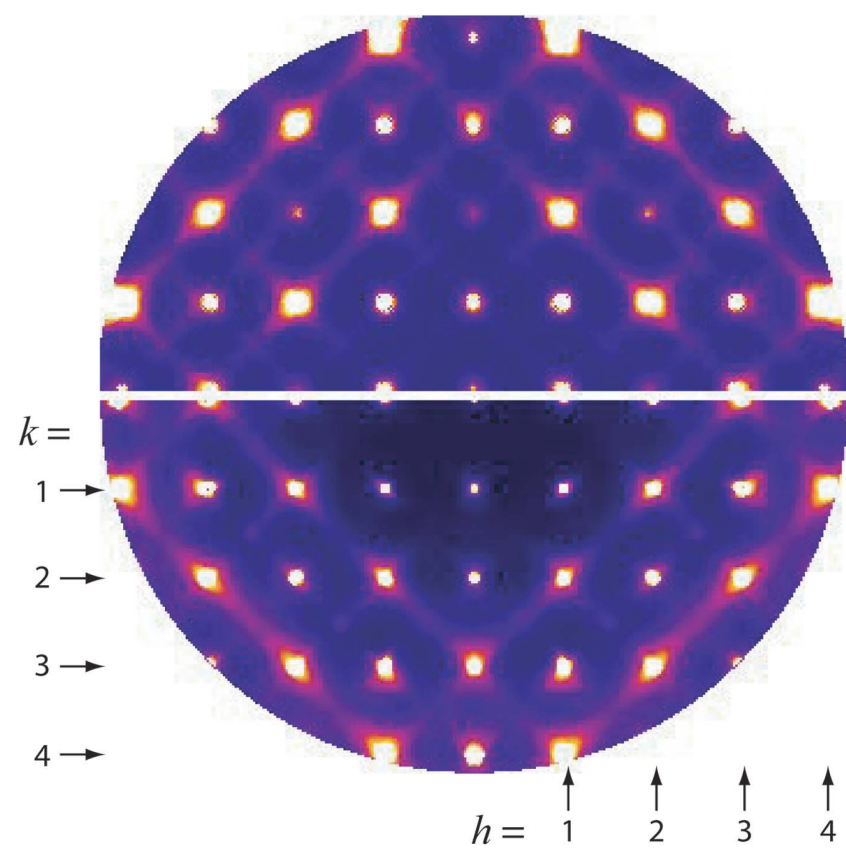

(b)

\section{Figure 5}

Calculated diffuse-scattering patterns for PZN (upper semicircles) compared with the corresponding observed neutron data (lower semicircles). ( $a$ ) $h k 0$ section. (b) $h k 1$ section. (c) $h k \frac{1}{2}$ section. (Note: the calculated patterns contain no actual Bragg peaks since the average structure is subtracted during the calculation.) 
envisaged to exist in the planar nanodomains. Fig. 6(a) shows the envisaged pattern of ion displacements in a domain normal to $[1 \overline{1} 0]$.

4.2.1. Ordering of $\mathbf{P b}$ displacements. The aim in building a MC simulation model was therefore to construct a potential that would tend to induce such planar domains normal to all six of the $\langle 110\rangle$ directions simultaneously, thus maintaining overall cubic symmetry. First it was assumed that at each $\mathrm{Pb}$ site the $\mathrm{Pb}$ ion is displaced away from the average site in one of the $12\langle 110\rangle$ vector directions by an amount defined by the 12 -state vector quantity $\Omega_{n}(i, j, k)$ whose components are defined by the variable $\delta$, as shown in Table 3. Here, $i, j, k$ are indices defining the primitive cubic lattice on which the $\mathrm{Pb}$ ions reside.

In order to try to form planar domains in which all $\mathrm{Pb}$ atoms within the domain are displaced in the same direction as shown in Fig. 6(a), we first build a simple potential which will induce correlations within planes normal to [1]0], both along [110] and along [001]. We specify that the energy of the $p$ th $\mathrm{Pb}$ ion due to interaction in the plane perpendicular to [110] is given by

$$
E_{[110]}^{p}=\sum_{\substack{[001] \text { in plane } \\ \text { neighbours }, q}} E_{1}^{p q}+\sum_{\substack{[110] \text { in plane } \\ \text { neighbours }, r}} E_{2}^{p r},
$$

where

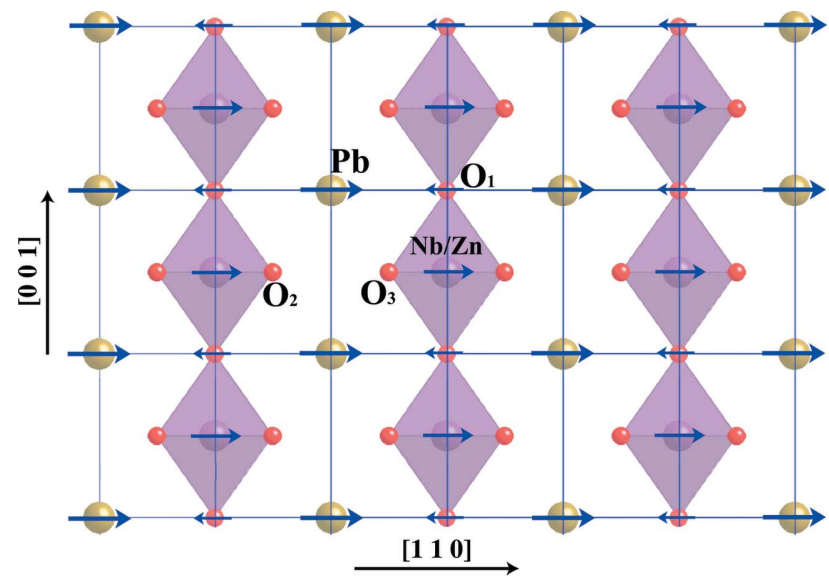

(a)

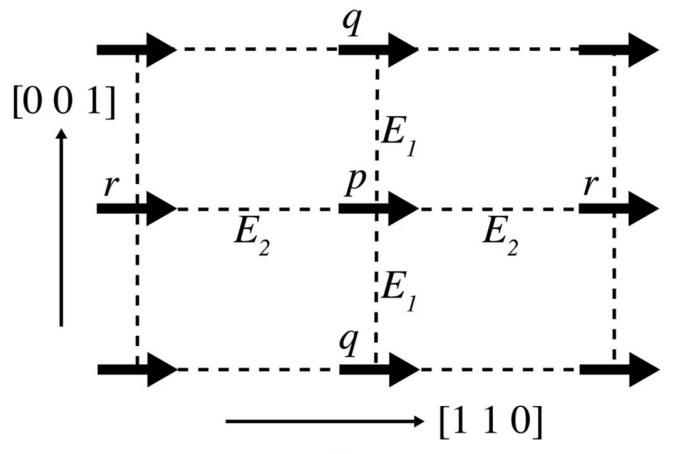

(b)

\section{Figure 6}

(a) View down [110] of the envisaged pattern of ion displacements within a planar nanodomain of PZN. (b) Definition of the energies $E_{1}$ and $E_{2}$ used in the MC energy.

$$
\begin{aligned}
& E_{1}^{p q}= \begin{cases}E_{1} & \text { if } \Omega^{p}\left\|\Omega^{q}\right\| \pm[110] \\
0 & \text { otherwise }\end{cases} \\
& E_{2}^{p r}= \begin{cases}E_{2} & \text { if } \Omega^{p}\left\|\Omega^{r}\right\| \pm[110] \\
0 & \text { otherwise. }\end{cases}
\end{aligned}
$$

$E_{1}^{p q}$ and $E_{2}^{p r}$ are energies defining the interaction between the vectors representing the $\mathrm{Pb}$ displacements on neighbouring sites as shown in Fig. $6(b) . E_{1}^{p q}$ is zero unless both the target $\mathrm{Pb}$ atom $(p)$ and its [001] neighbour $(q)$ are displaced along [110] (or both are displaced along [1 $\overline{1} 10]$ ), when it takes on value $E_{1}$. Similarly, $E_{2}^{p r}$ is zero unless both the target $\mathrm{Pb}$ atom $(p)$ and its [110] neighbour $(r)$ are displaced along [110] (or both are displaced along [110]). To get a total MC energy, additional sets of equations are used, related by symmetry to equation (3) and equation (4), for the other five $\langle 110\rangle$ directions. Then the total energy for atom $p$ is given by

$$
E_{\mathrm{total}}^{p}=E_{[011]}^{p}+E_{[01 \overline{1}]}^{p}+E_{[101]}^{p}+E_{[10 \overline{1}]}^{p}+E_{[110]}^{p}+E_{[1 \overline{1} 0]}^{p} .
$$

This form for MC energy is seen to contain only terms that lie within each $\{110\}$ plane of the nanodomains and it is implicit in this that the ordering in one such nanodomain is independent of the $\{110\}$ plane immediately above or below. Fig. 7 shows a small region of the ordering pattern obtained using equation (5). The two frames show successive layers normal to [1]10] in the three-dimensional structure. These patterns clearly show the formation of the desired nanodomains and that the domains in one layer are not related to those in the layers above or below, i.e. they are essentially thin plates.

4.2.2. Size-effect relaxation. For the $\mathrm{Pb}$ displacement ordering described above, the $\Omega_{n}(i, j, k)$ variables were considered to reside on the sites of a simple cubic lattice and so the atomic shifts defined in Table 3 are the only departures from the average site positions. However, it might be expected that on any given site the shifts will be modified depending on

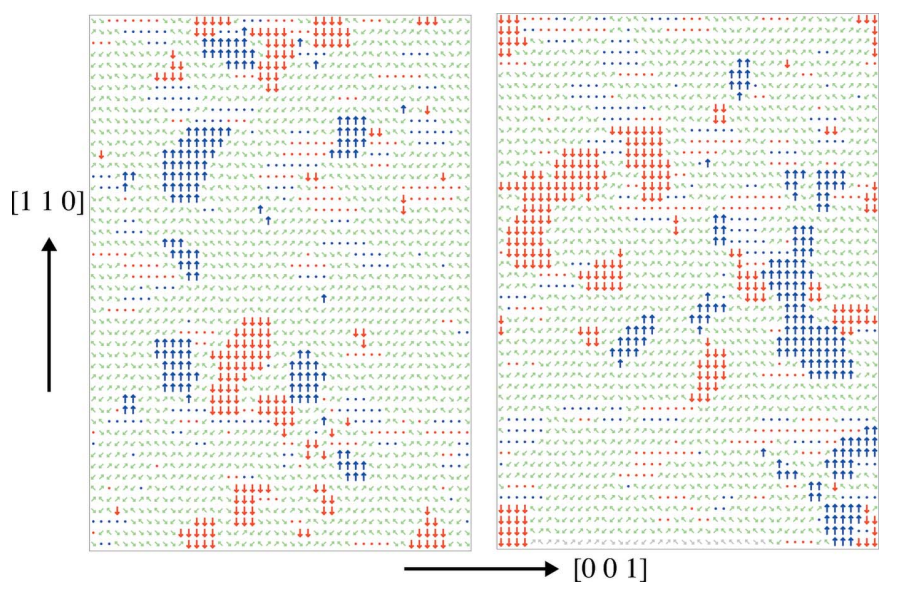

Figure 7

View down [110] of the ordering of the $\mathrm{Pb}$ displacement vectors within two successive layers within the three-dimensional structure. The red and blue arrows indicate displacement vectors in the plane. Green vectors are ones inclined to the plane. Red and blue dots are vectors normal to the plane. 
the values of $\Omega_{n}(i, j, k)$ values for the neighbouring sites, i.e. there will be additional local 'size-effect' relaxation shifts. These were introduced in a second stage of MC simulation in which the algorithm simply tried to increase the length of the $\langle 110\rangle$ vectors connecting two $\mathrm{Pb}$ ions if their displacements were opposed and similarly tried to decrease them if they were aligned. This was achieved using a MC energy of the form

$$
E_{\text {size }}=\sum_{\substack{\langle 110\rangle \\ \text { vectors }}}\left[d_{m, n}-\sqrt{2} a_{0}\left(1+\varepsilon_{m, n}\right)\right]^{2}
$$

Here $\varepsilon_{m, n}$ is a size-effect parameter.

4.2.3. Inclusion of other ions. As explained earlier, to account for the systematic extinction in the $(h k 1)$ section of the diffuse rods for $h+k=n$, with $n$ even, and the fact that in the $(h k 0)$ section there is no such extinction, it is necessary that the $B$-site cation ( $\mathrm{Nb}$ or $\mathrm{Zn}$ ) is displaced in phase with the $\mathrm{Pb}$ displacements and the $\mathrm{O} 1$ atom is displaced out of phase (as shown in Fig. 6a). In the current model, these two effects were achieved by inserting these ions into the structure in a deterministic way from the coordinates of the $\mathrm{Pb}$ ions resulting from the $\mathrm{MC}$ simulation. The $\mathrm{Nb} / \mathrm{Zn}$ ion coordinates were determined from the mean position of the eight surrounding $\mathrm{Pb}$-ion coordinates. The $\mathrm{O} 1$ atom shift along the vector between two $\mathrm{Pb}$ sites was determined from the positions of these $\mathrm{Pb}$ ions. See Welberry et al. (2006) for further details.

In order to account for the second set of diffuse peaks in the $h k \frac{1}{2}$ section (Fig. $5 c$ ), it is necessary to include local occupancy ordering of the $B$-site cations. This was achieved in a further stage of MC simulation in which the following energy was used:

$$
E_{B \text { site }}=\sum_{\substack{\text { all }\langle 100\rangle \\ \text { vectors }}} J \sigma_{m} \sigma_{n}
$$

Here $\sigma_{m}$ and $\sigma_{n}$ are $(+1 /-1)$ Ising spin variables at sites $m$ and $n$, respectively, on the primitive cubic $B$-site lattice. In the final model, antiferromagnetic ordering close to maximal for 2:1 stoichiometry was achieved by assigning a large positive value for $J / k T$. See Welberry et al. (2006) for further details.

4.2.4. Results. Diffraction patterns calculated from the final model outlined above are shown in Fig. 5 in comparison with the observed neutron data. Although the model, comprising as it does three different stages of MC simulation, may appear quite complex, it is in fact very simple since it contains very few adjustable parameters. First is the magnitude of $\delta$ defining the basic $\mathrm{Pb}$ shift. Second are the values of the ordering energies, $E_{1}$ and $E_{2}$ in equation (3), and finally the magnitude of the size-effect parameter, $\varepsilon_{m, n}$, in equation (6).

This relatively simple model is able to explain all the key features present in the diffuse scattering - a strong indication of its fundamental soundness. The model has led to the conclusion that the $\mathrm{Pb}$ displacements are directed along $\langle 110\rangle$ and that these form two-dimensional thin plate-like domains of aligned $\mathrm{Pb}$ displacements normal to each of the six $\langle 110\rangle$ directions, but it has also provided insight into the mechanism for domain growth in response to, for example, the imposition of an external electric field. The model gives clear insight into how a given nanopolar domain can grow (or shrink) by one unit cell at a time simply by an individual $\mathrm{Pb}$ atom adjacent to the domain boundary locally reorienting itself and switching from one $\langle 110\rangle$-type displacement direction to another. Although this will be accompanied by local relaxation, it does not require diffusion or long-range cooperative motion, and thus is of relatively low energy, contributing to the properties of this relaxor material.

The azimuthal variation of diffuse rod intensity is as close to a direct observation of the direction of the $\mathrm{Pb}$ displacements as is possible to find - far more direct than anything that can be inferred from Bragg scattering or spectroscopy. However, very recently, an alternative explanation of the diffuse scattering in relaxors such as PZN has been put forward (Pasciak et al., 2007). They postulate that the observed diffuse rods derive not from planar nanodomains as described above but from planar interfaces between three-dimensional domains. Further, the azimuthal variation of intensity in the rods is seen by them to derive from the relative displacements of $\mathrm{Pb}$ ions in neighbouring domains rather than their absolute direction. Further detailed quantitative analysis using this model is required to ascertain whether it can provide a better fit to the observed data than the current model.

\section{Discussion}

Although the analysis of diffuse scattering has lagged well behind the development of conventional crystallography, much has, nevertheless, been achieved since the first papers were published in Acta Crystallographica 60 years ago. With the advent of synchrotron sources and electronic area detectors, the collection of high-quality three-dimensional diffusescattering data is now relatively straightforward, although the low signal level still presents some problems not experienced in the measurement of Bragg scattering.

Similarly, the advent of fast and inexpensive computers over the last 15 years or so has led to the development of computer simulation methods that provide powerful tools for the interpretation and analysis of diffuse scattering. While conventional crystallography benefited from the fact that the same basic methods could be applied for all crystalline materials regardless of their complexity, the sheer diversity of different types of physical effects that give rise to diffuse scattering was hitherto the largest impediment to a universal method of analysis. With the development of computersimulation methods, there is, for the first time, the possibility of having a single general method that can be used for any diffuse-scattering problem in any type of system.

Such methods have already been used to study a diverse range of problems using diffuse-scattering data, e.g. stacking faults and columnar defects in zeolites (Campbell et al., 2004), defect clustering and local strain in oxide ceramics (Welberry \& Christy, 1997), orientational disorder and channel distortions in inclusion compounds (Welberry \& Mayo, 1996), coupled inter- and intramolecular motions in crystals of flexible molecules (Welberry et al., 2001), multisite correlations in transition-metal oxyfluorides (Withers et al., 2003), 
and size-effect strains in quasicrystals (Welberry \& Honal, 2002). As computers get ever faster, it is expected that current simulation methods will become increasingly powerful and applicable to a wider and wider range of problems and materials and provide results in increasingly fine detail.

The two examples that have been outlined in this paper serve to illustrate the kind of result that can currently be achieved in two quite different kinds of system and the sort of information that diffuse scattering can give that cannot be obtained from Bragg scattering. It is still true to say that, although in each case a satisfactory model was obtained that gave very useful insights into the detailed structural behaviour, each model is still amenable to further development. In the case of PZN, the way in which the non- $\mathrm{Pb}$ ions were added was grossly over-simplified and work is in progress to improve the model in this regard. The current agreement with the data suggests that significant further information can be extracted from the current data. For the case of PCNB, too, there is still room for improvement. In particular, the assumption of random occupancy of each molecular site by one of the six basic orientations is clearly only a first (albeit seemingly good) approximation. As the simulation methodology improves, this kind of finer detail may be accessible.

In this regard, there are many similarities between the current state of diffuse-scattering analysis and that of the embryonic conventional crystallography of 60 years ago. Full three-dimensional data are now available and the models that are being used are also fully three dimensional. However, analysis of these data is generally still confined to the use of a small number of (well chosen) planar subsets of the data. It is clear that, were it feasible for full three-dimensional data to be used in the analysis, great benefits would ensue in terms of the fine detail that could be resolved, just as occurred in conventional crystallography. At this point in time, with the power of computers having increased by a factor of $\sim 1000$ in each of the last four decades and with the prospects of this continuing at least for another decade (Moore's law), it seems likely that such three-dimensional analyses should be feasible in the not too distant future. The analysis of diffuse scattering thus appears poised to enter a period of expansion and exploitation, analogous to that which occurred for Bragg scattering 50 to 60 years ago.

The support of the Australian Research Council, the Australian Partnership for Advanced Computing, the Australian Access to Major Research Facilities Program and the Australian Synchrotron Research Program are gratefully acknowledged. Use of the Advanced Photon Source was supported by the US Department of Energy, Office of Science, Office of Basic Energy Sciences, under Contract No. DEAC02-06CH11357. DJG gratefully acknowledges the support of the Australian Institute of Nuclear Science and Engineering.

\section{References}

Abrahams, S. C. \& Robertson, J. M. (1948). Acta Cryst. 1, 252-259.

Allen, F. H. (2002). Acta Cryst. B58, 380-388.

Berman, H. M., Westbrook, J., Feng, Z., Gilliland, G., Bhat, T. N., Weissig, H., Shindyalov, I. N. \& Bourne, P. E. (2000). Nucleic Acids Res. 28, 235-242.

Bing, Y. H., Bokov, A. A., Ye, Z. G., Noheda, B. \& Shirane, G. (2005). J. Phys. Condens. Matter, 17, 2493-2507.

Borie, B. \& Sparks, C. J. Jr (1971). Acta Cryst. A27, 198-201.

Broomhead, J. M. \& Nicol, A. D. I. (1948). Acta Cryst. 1, 88-92.

Burns, G. \& Dacol, F. H. (1983). Phys. Rev. B, 28, 2527-2530.

Campbell, B. J., Welberry, T. R., Broach, R. W., Hong, H. \& Cheetham, A. K. (2004). J. Appl. Cryst. 37, 187-192.

Cole, J. M., McIntyre, G. J., Thomas, L. H. \& Wilson, C. C. (2007). Personal communication.

Cole, J. M., Wilson, C. C., Howard, J. A. K. \& Cruickshank, F. R. (2000). Acta Cryst. B56, 1085-1093.

Cross, L. E. (1987). Ferroelectrics, 76, 241-267.

Dmowski, W., Akbas, M. K., Davies, P. K. \& Egami, T. J. (2000). Phys. Chem. Solids, 61, 229-237.

Estermann, M. \& Steurer, W. (1998). Phase Transit. 67, 165-195.

Flack, H. (1970). Philos. Trans. R. Soc. London Ser. A, 266, 559-591.

Garrido, J. (1948). Acta Cryst. 1, 3-4.

Georgopoulos, P. \& Cohen, J. B. (1977). J. Phys. Colloque, 38, 191-196.

Glazer, A. (1970). Philos. Trans. R. Soc. London Ser. A, 266, 593-639.

Guinier, A. (1963). X-ray Diffraction in Crystals, Imperfect Crystals and Amorphous Bodies. San Francisco: W. H. Freeman and Co.

Guinier, A. \& Griffoul, R. (1948). Acta Cryst. 1, 188-193.

Hall, P. G. \& Horsfall, G. S. (1973). J. Chem. Soc. Faraday Trans 2, 69, 1071-1077.

Jerslev, B. (1948). Acta Cryst. 1, 21-27.

Lonsdale, K. (1948a). Crystals and X-rays. London: G. Bell \& Sons.

Lonsdale, K. (1948b). Acta Cryst. 1, 12-20.

Park, S. E. \& Shrout, T. R. (1997). J. Appl. Phys. 82, 1804-1811.

Pasciak, M., Wolcyrz, M. \& Pietraszko, A. (2007). Phys. Rev. B, 76, 014117.

Thomas, L. H. (2007). PhD thesis, University of Cambridge, UK.

Thomas, L. H., Welberry, T. R., Goossens, D. J., Heerdegen, A. P., Gutmann, M. J., Teat, S. J., Lee, P. L., Wilson, C. C. \& Cole, J. M. (2007). Acta Cryst. B63, 663-673.

Tibballs, J. E. (1975). J. Appl. Cryst. 8, 111-114.

Weber, T. \& Bürgi, H.-B. (2002). Acta Cryst. A58, 526-540.

Weber, T., Estermann, M. A. \& Bürgi, H.-B. (2001). Acta Cryst. B57, 579-590.

Welberry, T. R. (2001). Acta Cryst. A57, 244-255.

Welberry, T. R. (2004). Diffuse X-ray Scattering and Models of Disorder. IUCr Monographs on Crystallography. Oxford University Press.

Welberry, T. R. \& Christy, A. G. (1997). Phys. Chem. Miner. 24, 24-38.

Welberry, T. R., Goossens, D. J., Edwards, A. J. \& David, W. I. F. (2001). Acta Cryst. A57, 101-109.

Welberry, T. R., Goossens, D. J. \& Gutmann, M. J. (2006). Phys. Rev. $B, 74,224108$.

Welberry, T. R., Goossens, D. J., Heerdegen, A. P. \& Lee, P. L. (2005). Z. Kristallogr. 222, 1052-1058.

Welberry, T. R., Gutmann, M. J., Woo, H., Goossens, D. J., Xu, G., Stock, C., Chen, W. \& Ye, Z.-G. (2005). J. Appl. Cryst. 38, 639-647. Welberry, T. R. \& Honal, M. (2002). Z. Kristallogr. 217, 1-4.

Welberry, T. R. \& Mayo, S. C. (1996). J. Appl. Cryst. 29, 353-364.

Withers, R. L., Welberry, T. R., Brink, F. J. \& Norén, L. (2003). J. Solid State Chem. 170, 211-220.

Woodward, P. M. \& Baba-Kishi, K. Z. (2002). J. Appl. Cryst. 35, 233-242.

Ye, Z. G., Dong, M. \& Zang, I. (1999). Ferroelectrics, 229, 223-232. 